\title{
Multiphysics modeling in support of ultrasonic image development: integration of fluid-structure interaction simulations and Field II applied to the carotid artery
}

\author{
Abigail Swillens \\ Gianluca De Santis \\ Patrick Segers \\ IBiTech-bioMMeda
}

Ghent University, Ghent, Belgium

Email: abigail.swillens@ugent.be

\author{
Lasse Lovstakken \\ Department of Circulation and Medical Imaging \\ NTNU, Trondheim, Norway \\ St. Olavs University Hospital, Trondheim, Norway
}

\author{
Joris Degroote \\ Jan Vierendeels \\ Department of flow, heat \\ and combustion mechanics
}

Ghent University, Ghent, Belgium

\begin{abstract}
Previously, we proposed a multiphysics model coupling computational fluid dynamics (CFD) and Field II, allowing assessment of the performance of current and new blood flow estimators (e.g. color flow imaging=CFI, PW Doppler, speckle tracking, vector Doppler) in the carotid artery against ground truth information retrieved from CFD. Important limitations however were the rigid walls and the absence of the arterial wall and surrounding tissue in the simulations. The aim of this study was to improve and expand the model to a more realistic setup of a distensible carotid artery embedded in surrounding tissue. For this purpose, we integrated fluid-structure interaction (FSI) simulations with an ultrasound simulator (Field II), which allows comparison of the ultrasound (US) images with the input data from FSI. Field II represents tissue as random points on which ultrasound waves reflect and whose position can be updated based on the flow field and vessel wall deformation from FSI. We simulated the RF-signal of a patient-specific carotid bifurcation, including the blood pool as well as the vessel wall and surrounding tissue. Realism of the multiphysics model was demonstrated with duplex images.
\end{abstract}

\section{INTRODUCTION}

Ultrasound imaging of the carotid artery is a common procedure when screening for cardiovascular disease, as the vessel is particularly prone to atherosclerosis and easily accessible with ultrasound probes. However, to reveal abnormalities in carotid flow and wall deformation with increased sensitivity and specificity, improved imaging modalities are desired. Since invitro and in-vivo testing of new imaging algorithms are often too simplified or lack ground truth information, ultrasonic image simulation based on fully known and realistic vascular behavior can be expected to support image development.

In this context, we developed a multiphysical simulation environment which integrates advanced numerical methods to calculate complex flow patterns and mechanical deformations on the one hand with an ultrasonic simulator on the other hand. For the ultrasonic image modeling, we relied on the Field II-software [1], [2], which can simulate with great scanning flexibility images of arbitrary tissue (i.e. both blood and arterial wall), by representing tissue as an ensemble of point scatterers on which the ultrasound waves reflect. In a first phase, we coupled computational fluid dynamics (CFD) with Field II, allowing simulation of radiofrequency (RF)-data from realistic and complex flow fields, by moving the scatterers according to the spatially and temporally interpolated velocity fields obtained from CFD. We demonstrated the realism of the simulation environment with color flow imaging and pulsed wave Doppler examples in the carotid artery [3] and by validating the simulation strategy on an in-vitro flow phantom of the carotid bifurcation [4]. Fundamental limitations of this approach were the absence of the vessel wall signal and the rigid vessel walls.

In a next phase, we integrated fluid-structure interaction (FSI) simulations with the ultrasound simulator [5], which allowed to simultaneously assess the complex flow field and vessel wall deformation by coupling the numerical solution of a dedicated flow solver and structural solver. Hence, an FSI-Field II integration offers the possibility to simultaneously simulate the RF-signal of the blood pool and the moving vessel wall. This, however, requires a much more complex coupling methodology (derivation of the scatterer displacement) compared to the CFD-Field II coupling, due to the temporally varying fluid volume and the complex layered vessel wall, as explained in [5]. We demonstrated this FSI-Field II coupling in a 3D straight tube, representative of the common carotid artery [5].

In this work, we further advance the realism of the synthetic vascular imaging set-up with the simulation of the 3D blood flow and arterial mechanics of a patient-specific carotid bifurcation model. The model includes the tissue surrounding the vascular wall, which is a stabilizing factor for the fluidstructure interaction simulation but also results in a more realistic ultrasonic visualization of the arterial territory. The extensive methodology behind the FSI-Field II coupling will be briefly discussed, before demonstrating the realism of our 


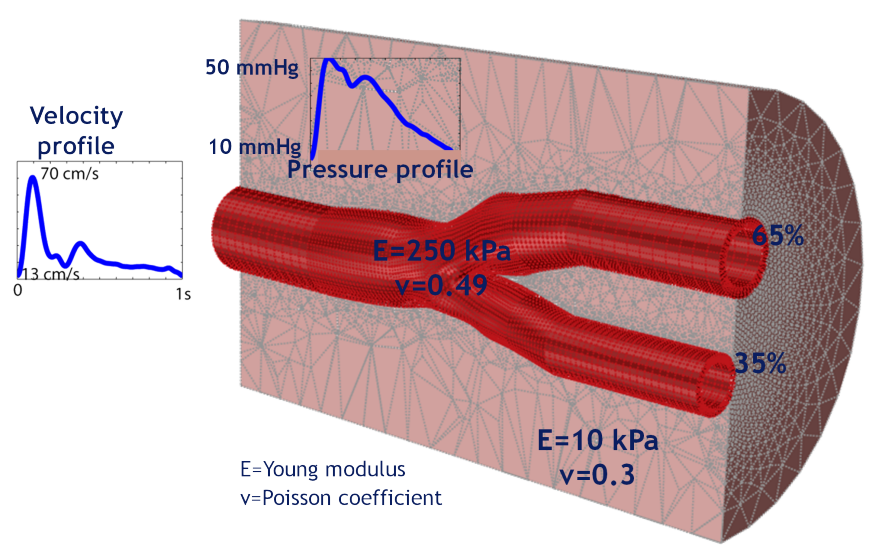

Fig. 1. Setup of the FSI-simulations

multiphysics modeling with simulated duplex images.

\section{Methods}

\section{A. FSI-simulations}

1) Numerical approach: A partitioned FSI-approach was followed, i.e. the equations for the flow and structural domain were solved separately with a dedicated flow (Fluent 12.0.16, Ansys, Canonsburg, PA, USA) and structural solver (Abaqus 6.7, Simulia,Inc., Providence, RI, USA). The solutions for the fluid and structural domain were coupled using in-house code (Tango) with Dirichlet-Neumann partitioning (the flow problem is solved for a given displacement of the fluidstructure interface, and the structural problem is solved for a given stress distribution on the wet side of the structure). A converged solution for both the fluid and structural equations (and coupling conditions) was found by performing coupling iterations between both solvers, until equilibrium between the fluid and structure was reached. To enhance convergence of the coupling iterations, an Interface Quasi-Newton (IQN) method was used [6]. Note that the fluid and structural domain have inherently different grid formulations, which was solved by using an Arbitrary Lagrangian Eulerian (ALE) method for the fluid domain. For more information on the IQN and ALE method, we refer to [5].

2) (Meshing the) carotid geometry: The 3D geometry was reconstructed from CT-scans of a stenosed carotid bifurcation of an 83-year old volunteer. An in-house open-source software package (Pyformex, pyformex.org) was used to construct a computational grid for the vascular domain. This allowed to create a layered mesh of the vascular wall (to some extent mimicking the intima-media-adventitia layers), consisting of 31680 first order hexahedral elements and refined near the lumen. Pyformex was subsequently used to mesh the vascular lumen with hexahedrons, resulting in 87522 elements and a matching grid at the fluid-structure interface, again refined near the vascular wall.

The structural domain was further expanded to also include the tissue surrounding the vascular wall. The carotid artery was embedded in a cylinder with a radius of $2 \mathrm{~cm}$. A tetrahedral element type was chosen for the tissue volume (62 717 elements). To ensure continuity of the hexahedral and tetrahedral meshes at the wall-tissue interface, the quadrilaterals at the outer surface of the vascular wall were split into triangles to match with the tetrahedrons of the tissue domain.

3) FSI-setup: Fluid domain: At the in- and outlets of the carotid geometry, we imposed physiologically realistic boundary conditions. We measured a velocity profile with pulsed wave Doppler (12L linear array vascular probe, GE Medical Systems, Milwaukee, WI, USA) in the common carotid of a healthy volunteer, which was further applied as a mass flow inlet condition. Outflow percentages were imposed at the outlets $(35 \%$ at the external and $65 \%$ at the internal carotid). A non-invasively measured pressure (varying in time, with a pulse pressure of $40 \mathrm{mmHg}$ ) was further imposed. As such, when transferring the interface stress to the structural solver, a realistic pressure value was imposed on the wet side of the structure. Blood was modeled as a Newtonian liquid with a viscosity of $3.5 \mathrm{mPa} \cdot \mathrm{s}$ and a density of $1050 \mathrm{~kg} / \mathrm{m}^{3}$.

Solid domain: Assuming that the mechanical properties of the vessel wall material can be linearized around the operating pressure, we modeled the vessel wall as a linear elastic material with Young's modulus of $250 \mathrm{kPa}$, density of 1200 $\mathrm{kg} / \mathrm{m}^{3}$ and Poisson modulus of 0.49 (nearly incompressible). The properties of the surrounding tissue were chosen to obtain a realistic distension degree for the chosen vessel elasticity: a Young's modulus of $10 \mathrm{kPa}$ and Poisson modulus of 0.3 . Longitudinal movement of the in- and outlets was prevented. We refer to fig. 1 for a complete overview of the simulation setup. The cardiac cycle of $1 \mathrm{~s}$ was divided into timesteps of $5 \mathrm{~ms}$.

\section{B. Ultrasound-simulations}

1) Field II: The Field II software [1], [2] was used to simulate the RF-signals from the fluid and structural domain. The RF-signals can be simulated by modeling tissue as a distribution of (random) point scatterers, whose position can be updated for each simulated ultrasound beam. By moving the scatterers according to flow fields and wall deformations obtained from FSI, imaging algorithms can be studied in complex conditions [3], [5].

Fluid phantom: In [5], we described a method to generate scatterers during Field II simulations based on CFDcalculations with rigid walls. 3D spatial interpolation was performed to transform the CFD velocity vectors to the random scatterer cloud, and temporal interpolation of the CFD velocities was required due to the large disparity in the ultrasound and CFD timescales (63 $\mu$ s versus $5 \mathrm{~ms}$ for the simulated color flow imaging application). For distensible fluid geometries, straightforward linear temporal interpolation of the velocity fields is however not possible. This is illustrated in fig. 2 , if one considers the position of the red point at time point $T_{F S I, 1}$ of the FSI-calculation and the same absolute position of this point at the next FSI timestep $T_{F S I, 2}$. In case of a decreasing fluid volume, this point ends up outside the fluid volume at $T_{F S I, 2}$ (white point in fig.2). In case of increasing 


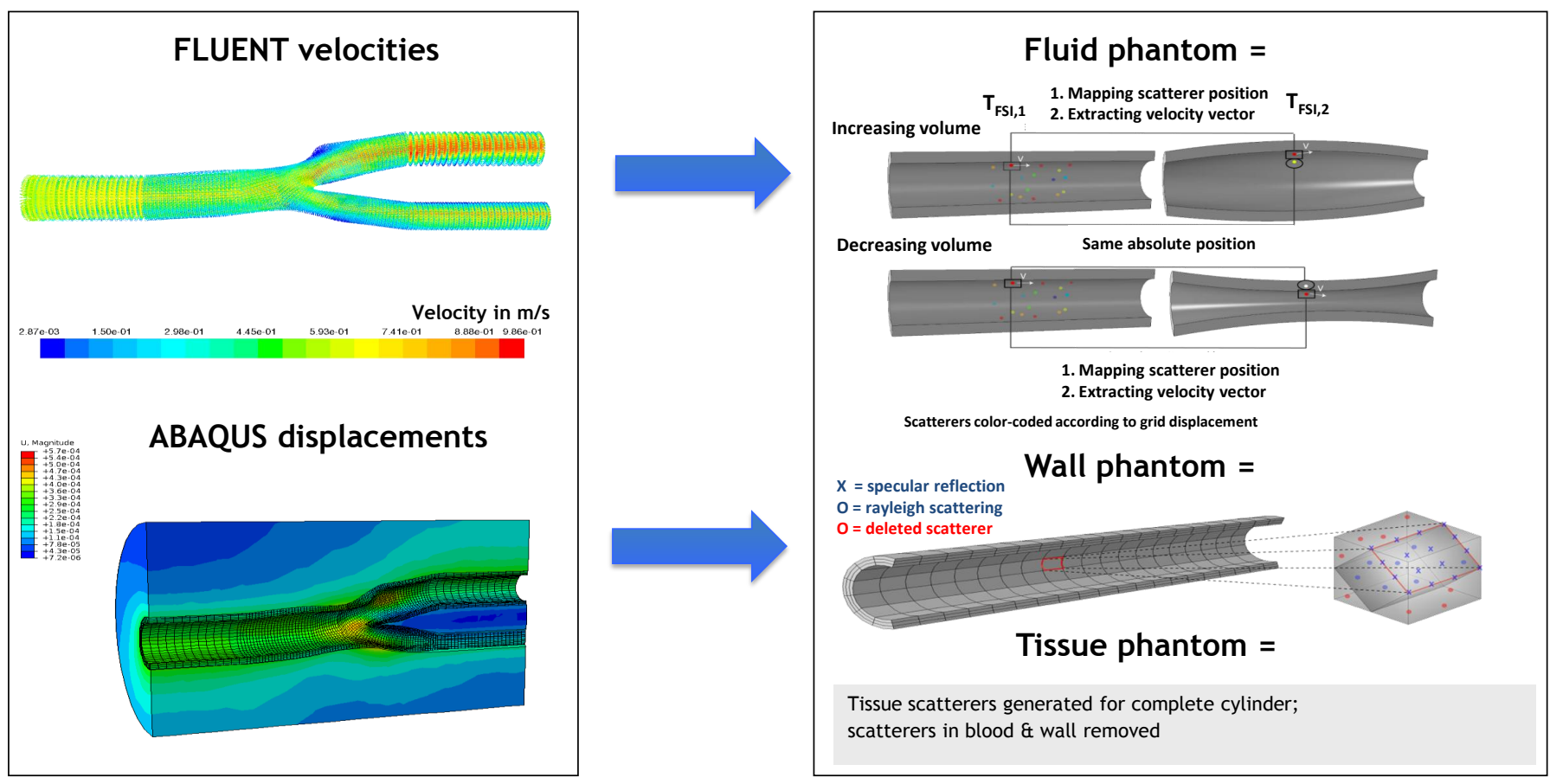

Fig. 2. Strategy behind integrating fluid-structure interaction simulations and Field II

fluid volume, the point ends up further from the vessel wall at $T_{F S I, 2}$ compared to $T_{F S I, 1}$ (yellow point in fig.2). Hence, temporal interpolation between the same absolute positions at different FSI-timesteps is incorrect. Complex mathematical methods exist to derive velocity fields for changing fluid domains at timesteps intermediate to the chosen FSI-timesteps. This, however, would be an impractical approach due to high computational times. We refer to [5] for further details on how the scatterers can be propagated using FSI simulation results accounting for the temporally varying fluid volume and the applied ALE grid formulation.

Wall phantom: To account for local changes in material (acoustic) properties, random point scatterers were generated for each element of the wall mesh. Further, as can be seen in echo images, the vessel wall also causes specular reflections due to transitions between different tissue types. These cannot be simulated but only mimicked in Field II, by placing scatterers in a structured fashion at the borders of the vessel wall (i.e. tissue/vessel wall and vessel wall/blood). For more details on the coupling methodology for the wall, we again refer to [5].

Tissue phantom: To reduce computational times, scatterers were not generated for each mesh element of the tissue (as for the wall phantom), but for the complete cylinder surrounding the carotid artery, comprising the fluid, wall and tissue domain. Afterwards, scatterers created inside the arterial wall and fluid volume were removed.

2) Imaging setup: A linear array transducer was modeled and imaging parameters can be found in table 1 .

Duplex scanning: A duplex scan is the superposition of a color flow image (CFI) on a B-mode image. However, the image acquisition requirements of CFI and B-mode are inherently different due to dissimilar spatial and temporal resolution requirements, and therefore compromises have to be made. A $5 \mathrm{MHz}$ centre frequency was chosen for both image acquisitions, but the beam density was doubled for the B-mode imaging and the pulse length was increased for CFI from 1.5 to 4 pulse periods. To achieve these differing imaging properties, an interleaved scanning scheme was applied, switching between color flow and B-mode acquisitions. This resulted in a frame rate of $12 \mathrm{fps}$. The color flow velocities were estimated with the autocorrelation method for phase-shift estimation. The phantom was angled 70 degrees towards the ultrasound scanline to reduce flow transversal to the beam. To improve the CFI frame rate, we applied a beam interleaved

TABLE I

ULTRASOUND SET-UP PARAMETERS FOR DUPLEX SCANNING AND WALL TRACKING

\begin{tabular}{|l|l|}
\hline & CFI/B-mode \\
\hline center frequency & $5 \mathrm{MHz}$ \\
\hline number of elements & 192 \\
\hline element pitch & $0.25 \mathrm{~mm}$ \\
\hline element height & $6 \mathrm{~mm}$ \\
\hline transmit focus $\mathrm{Az}$ & $2 \mathrm{~cm}$ \\
\hline dynamic receive focusing & yes \\
\hline expanding aperture & yes \\
\hline excitation & sinusoidal \\
\hline pulseperiods & 4 \\
\hline$P R F_{\text {max }}$ & $16 \mathrm{kHz}$ \\
\hline PRF & $4 \mathrm{kHz} / 16 \mathrm{kHz}$ \\
\hline packetsize & $10 / 1$ \\
\hline
\end{tabular}



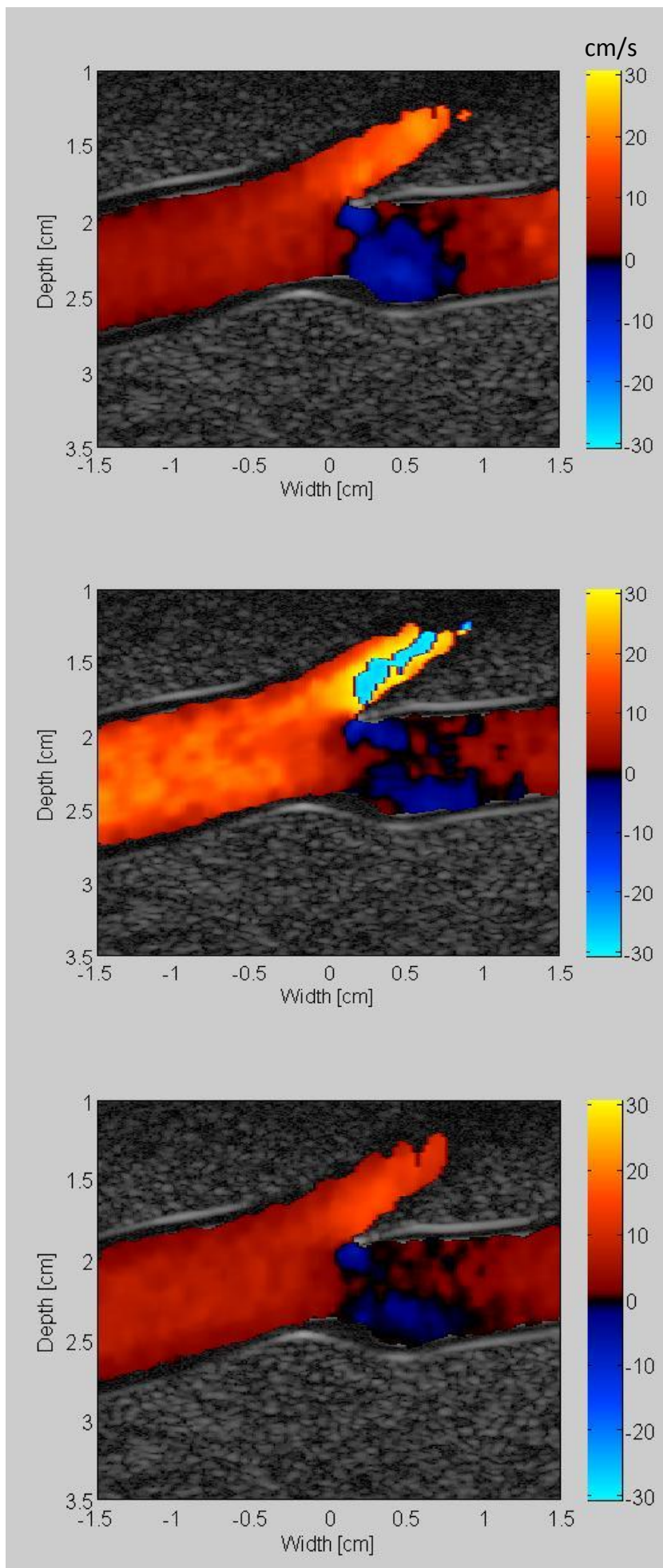

Fig. 3. Duplex images from multiphysics simulations:systolic acceleration, deceleration and diastole

acquisition scheme, typically used when the Doppler PRF (as determined by the imaged velocity range) is chosen lower than the maximal possible $P R F_{\max }$ (as determined by the imaging depth). We chose a setup with a PRF of $4 \mathrm{kHz}$, a $P R F_{\max }$ of $16 \mathrm{kHz}$, resulting in an interleave groupsize of 4 beams.

\section{RESULTS}

\section{A. Duplex scanning}

Duplex images are shown during systolic acceleration and deceleration, and diastole in fig. 3. Complex flow patterns are present throughout the cardiac cycle, and specular reflections are apparent when perpendicularly insonifying the blood vessel. This is particularly visible for the disappearing specular reflections in the external carotid artery as can be seen in the B-mode part of the image on fig.3.

\section{DISCUSSION AND CONCLUSION}

We successfully expanded our multiphysics simulation tool to the 3D deforming carotid artery, accounting for (the RFsignal from) the surrounding tissue and hence increasing the realism of the simulations. However, we should note that although our multiphysics simulations offer the opportunity to compare ultrasonic measurements with a ground truth, limitations exist both at the level of the FSI-simulations as well as the ultrasound model. While we applied an advanced FSI-code, resulting in a strongly coupled solution for the flow and structure, the imposed boundary conditions (both for the structure and fluid) and the assumed material model can be improved. Also the ultrasonic simulator shows limitations, as it is restricted to linear wave propagation and does not allow modeling complex wave phenomena typically encountered in medical scanning (e.g. reverberations and aberrations). Further, Field II does not take into account the true scatterer nature of the tissue (e.g. red blood cells are represented by points instead of discs, showing no inertia, no interaction and no frequency dependent scattering).

\section{REFERENCES}

[1] J. A. Jensen, "Field: A program for simulating ultrasound systems," Medical and Biological Engineering and Computing, vol. 34, pp. 351352, 1996.

[2] J. A. Jensen and N. B. Svendsen, "Calculation of pressure fields from arbitrarily shaped, apodized, and excited ultrasound transducers," IEEE transactions on Ultrasonics, Ferroelectrics, and Frequency control, vol. 39, no. 2, pp. 262-267, 1992.

[3] A. Swillens, L. Lovstakken, J. Kips, H. Torp, and P. Segers, "Ultrasound simulation of complex flow velocity fields based on computational fluid dynamics," IEEE Transactions on Ultrasonics, Ferroelectrics and Frequency Control, vol. 56, no. 3, pp. 546-556, 2009.

[4] A. Swillens, T. De Schryver, L. Lovstakken, H. Torp, and P. Segers, "Assessment of numerical simulation strategies for ultrasonic color blood flow imaging, based on a computer and experimental model of the carotid artery," Annals of Biomedical Engineering, vol. 37, no. 11, pp. 21882199, 2009.

[5] A. Swillens, J. Degroote, J. Vierendeels, L. Lovstakken, and P. Segers, "A simulation environment for validating ultrasonic blood flow and vessel wall imaging based on fluid-structure interaction simulations: ultrasonic assessment of arterial distension and wall shear rate.," Medical Physics, vol. 37, no. 8, pp. 4318-4330, 2010.

[6] J. Degroote, K. J. Bathe, and J.Vierendeels, "Performance of a new partitioned procedure versus a monolithic procedure in fluid-structure interaction," Computers \& Structures, vol. 87, p. 793801, 2009. 